\title{
Molecular Characterization and Phylogenetic Analysis of Orf Virus Isolated From Goats in Sokoto Metropolis, Nigeria
}

*Nafiu Lawal ${ }^{1,2}$, Mubarak Ibrahim ${ }^{1}$, Dauda Ayomide Onawala ${ }^{1}$, Muhammad Bashir Bello ${ }^{1,2,3}$, Rabiu Muhammad Aliyu ${ }^{1}$ Yushau Shuaibu Baraya ${ }^{4}$, Abdullahi Aliyu ${ }^{4}$, Aliyu Musawa Ibrahim ${ }^{5}$ and Aliyu Sa' adu ${ }^{6}$

Aim: Despite the endemic nature of contagious ecthyma in Nigeria, there is limited report on the molecular characterization of the isolates responsible for disease outbreaks. The aim of this study was to molecularly characterize ORFV isolated from clinical infections in goats in Sokoto metropolis.

Materials and Methods: Seronegative embryonated chicken eggs were used to isolate ORFV via the chorio allantoic membrane (CAM) route according to the established protocol. Viral DNA was extracted from infected CAM and the full coding region of B2L gene was amplified by PCR and subsequently sequenced by Sanger's method. The nucleotide sequence results were blasted for identification and phylogenetically analyzed using MEGA and Bioedit softwares.

Results and Discussion: The results showed that B2L gene sequences of the ORFV UDUS/01/19/More strain showed slight variability (96-98.7\%) with the reference sequences. Our isolate clustered within the same clade with Korean strain signifying a close genetic relationship. Unique amino acid substitutions were noted in our isolate when compared with other references. This is arguably the first genetic characterisation of B2L gene of ORFV circulating in Nigeria.

Conclusion: Our study has provided in sight into the genetic diversity of ORFV in the study area. This is crucial for the design of effective vaccines against the disease which are currently lacking in the country.

Keywords: ORFV, PCR, Sanger method, B2L gene, Phylogenetic analysis

\section{Background}

Contagious ecthyma (CE) is a highly contagious viral disease of small ruminants such as sheep and goats (1) that occasionally affects camels (2) and wild ruminants with huge economic impact on the livestock industry (2-6). Clinically, the disease is associated with skin lesions such as erythema, macules, papules, vesicles, 
39 pustules and crusts on the lips, tongue, teat, nose, hooves and other

40 parts of the body (6) especially in young lambs and kids $(3,7)$. The 41 disease is generally self-limiting, however, secondary bacterial 42 infection may complicate the situation, causing in-appetence, severe 43 emaciation and death of the affected animals (1). Epidemiological 44 evidence indicated morbidity of $60 \%$ but the mortality is usually 45 low unless complicated by secondary bacterial infection (8) where it 46 can reach up to $10 \%$ and $93 \%$ in kids and lambs respectively (9) and 47 even $100 \%$ in adult goats (4). The disease is also of zoonotic 48 significance, causing ulcerative lesions or nodules on the hands of 49 high risk individuals such as veterinarians, butchers and other 50 animal handlers (10). Except in immunocompromised patients, 51 most human cases of contagious ecthyma are localised and heal 52 spontaneously (11).

53 The aetiology of CE is Orf virus (ORFV), a member of the genus 54 Parapoxvirus in the family Poxviridae (12). The genetic material of the 55 virus is a linear double stranded DNA (13) of $134-139 \mathrm{~kb}$ in size $56(3,13)$. It exhibits high GC content of about $66 \%$ (14) and is generally 57 organised into conserved central portion and variable terminal 58 regions (15). The central portion has a number of genes including 59 the B2L gene, that encodes the immunogenic major envelope 60 protein p42K $(3,4,16,17)$. This gene has been extensively used for 
61 molecular detection and diagnosis (18) as well as phylogenic

62 analyses of various ORFV isolates $(3,13,19)$.

63 Laboratory diagnosis of CE can be achieved using electron 64 microscopy, histopathology and serological tests such as 65 Fluorescent Antibody Technique (IFAT), Virus Neutralization Test 66 (VNT), Agar Gel Immunodiffusion (AGID) and Enzyme Linked 67 Immunosorbent Assay (ELISA) (1,3). Nowadays, confirmation of $68 \mathrm{CE}$ is achieved using polymerase chain reaction (PCR) which has 69 been shown to be highly specific and sensitive $(1,13,16,19)$. Using 70 PCR, sequencing and phylogenetic analysis, genetic characteristic 71 and diversity of ORFV has been described in many countries 72 around the world including China (5,19,20), Taiwan (13), Malaysia 73 (7), India (12), Uruguay (3) and few African countries such as 74 Tanzania (21), Ethiopia (17,22), Egypt (23,24), Gabon (25) and 75 Sudan (26,27). To date, outbreaks of CE in Nigeria are largely 76 reported based on clinical manifestation of the disease and PCR to 77 confirm cases $(1,2,4)$ but no literature on the molecular 78 characterization of the circulating ORFV isolates is currently 79 available in Nigeria as at the time of this study. Therefore, in the 80 present study we reported for the first time the isolation, molecular 81 detection and phylogenetic characterisation of ORFV obtained from 82 a flock of goats in Sokoto metropolis, North Western Nigeria. 
83

84

85

86

87

88

89

90

92

93

94

95

96

97

98

99

100

101

102

103

104

105

\section{Materials and methods}

\section{Sample collection, transport and processing}

Suspected outbreak of CE was reported in a goat farm located in More area, Sokoto metropolis $\left(13.0059^{\circ} \mathrm{N}, 5.2476^{\circ}\right.$ E) in May, 2019. On visitation to the farm, a flock of 30 goats consisting of Red Sokoto Goat (RSG) and crosses of RSG with West African Dwarf (WAD) was observed (8 males, 22 females). Five of the males were less than a year, while 3 were 2-3 years of age. The females on the other hand consisted of 10 adults (2-4 years) and 12 kids (less than a year). Physical examination of the two affected goats (2 year old male RSG-WAD cross and 3 year old female RSG) revealed scab lesions on the ears, lips, and nose (Figure 1). Two samples (1 and 2) involving thick brown scabs were scrapped on clean paper from the two affected goats and immediately transferred in a sterile sample container containing phosphate buffered saline (PBS, pH 7.2-7.4), placed on ice and immediately transported to the Central veterinary Research Laboratory, Usmanu Danfodiyo University Sokoto for analysis. After sample collecton, the two animals were isolated from the rest of the flock and were treated with long acting oxytetracycline $20 \%$ at $1 \mathrm{~mL} / 20 \mathrm{~kg}$ body weight to prevent secondary bacterial infection and the scrapped lesions were scrubbed with povidine iodine and sprayed with gentian violet to facilitate wound healing. 
106 The samples were homogenized in phosphate buffered saline (PBS)

107 using tissue homogeniser and centrifuged at $1000 \times g$ for 15 minutes 108 to harvest the supernatants for storage at $-20^{\circ} \mathrm{C}$ until needed for 109 further analysis.

110
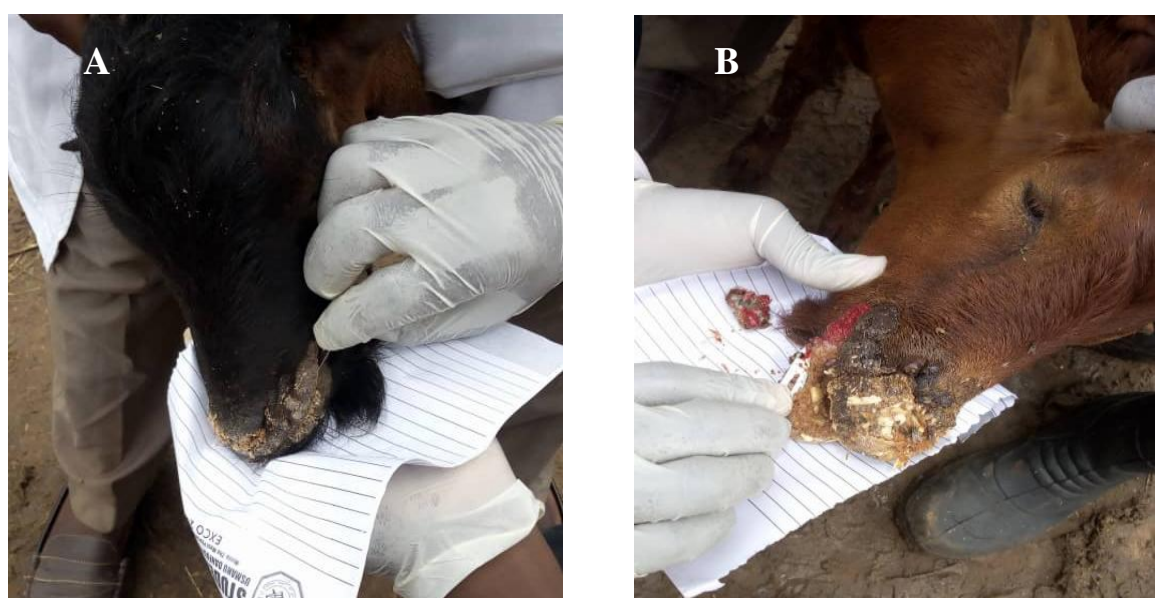

Figure 1: Sample collection from goats with suspected ORFV infection. Sample 1 from male RSG-WAD cross (Plate A) and sample 2 from female RSG (Plate B)

\section{Virus isolation}

Nine to eleven days old specific antibody free chicken embryonated eggs (CEE) were obtained from the Vaccine Research Division of the National Veterinary Research Institute Vom, Nigeria. The eggs were candled to ensure their viability and were later inoculated with $500 \mu \mathrm{L}$ each of the two prepared samples via the chorioallantoic membrane (CAM) route as described by (28), sealed and labelled appropriately. The eggs were incubated and observed daily for 5 to 7 days during which eggs with dead embryos were chilled at $4^{\circ} \mathrm{C}$. At the end of the incubation period, those embryos still alive were placed at $4^{\circ} \mathrm{C}$ overnight. Subsequently, the CAM was harvested and observed for the development of pock lesions before being stored at $-20^{\circ} \mathrm{C}$ until processed. 
124

125 Infected CAM harvested from the eggs inoculated with the two 126 samples were used for genomic DNA extraction using DNA Mini 127 kit (QIAGEN). Briefly, about $250 \mathrm{mg}$ of CAM was homogenised 128 and placed in a $1.5 \mathrm{ml}$ micro centrifuge tube. Lysis buffer and 129 proteinase $\mathrm{K}$ were added followed by incubation at $56^{\circ} \mathrm{C}$ in a water 130 bath until complete lysis of the tissues occurs. DNA was then 131 extracted according to the manufacturer's instructions, eluted with 13250 ul elution buffer and stored at $-20^{\circ} \mathrm{C}$.

133 Polymerase chain reaction

134 The ORFVB2LF1 5'-TCCCTGAAGCCCTATTATTTTTGTG-3' and ORFVB2LR15'-GCTTGCGGGCGTTCGGACCTTC-3' specific 137 used to amplify the complete B2L gene of the ORFV with the aid of 138 Toptaq PCR mastermix (Qiagen) according to the manufacturer's 139 instructions. The mixture was briefly centrifuged and placed in the 140 thermocycler. Amplification was carried out using initial 141 denaturation at $94^{\circ} \mathrm{C}$ for $3 \mathrm{~min}, 35$ cycles of $94^{\circ} \mathrm{C}$ for $30 \mathrm{sec}, 60^{\circ} \mathrm{C}$ for $14230 \mathrm{sec}$ and $72^{\circ} \mathrm{C}$ for $1 \mathrm{~min}$. Final extension was performed at $72^{\circ} \mathrm{C}$ 143 for 5 min. The amplified products were then analyzed by 144 electrophoresis on a $1.5 \%$ agarose gel containing $0.5 \mathrm{ng} / \mathrm{ml}$ 145 ethidium bromide in TAE buffer. The amplicons were viewed using 146 a GelDoc imaging system (BioRad). 
147 DNA sequencing and phylogeny and evolutionary analysis

148 PCR positive samples were sent to Inqaba, South Africa for Sanger 149 sequencing. The sequencing company reported that only one of the 150 two samples passed quality control (QC) for downstream 151 sequencing. Since the two samples were obtained from the same 152 outbreak in the same flock, we asked the company to go ahead and 153 sequence the sample that passed QC as the isolates in the two 154 samples are highly likely to be the same. On receiving the result for 155 the single sample, the Sequence was trimmed and subjected to 156 BLAST similarity search using the BLASTN algorithm of the NCBI 157 database, to confirm the identity of the virus. Subsequently, the 158 obtained sequence was deposited in the GeneBank database with 159 accession no MT272780. Reference sequences were downloaded 160 and aligned with the sequence obtained in this study using 161 ClustalW in the MEGA7 software (30). Phylogenetic tree was 162 constructed using the Neighbour-joining method with 2000 163 bootstrap replicates using MEGA7. Evolutionary distances were 164 inferred based on Pair Wise Sequence Comparison between the 165 isolates obtained in this study and the reference sequences. 


\section{Results}

\section{Virus isolation}

168 Following the inoculation of the processed two scab materials into 169 the specific antibody free CEE, pathologic changes in form of small 170 grayish white foci (pock lesions) were observed on the harvested 171 CAM membranes. These changes were not observed in the mock 172 inoculated eggs. This signifies the successful presumptive isolation 173 and identification of the virus.

\section{$174 \quad$ PCR and Sequence Analysis}

175 The PCR amplification of the CAM homogenate from the two 176 samples yielded a product of the B2L gene fragment at the expected 177 band size of $1137 \mathrm{bp}$ when analyzed by gel electrophoresis (Figure 178 2). The PCR product of sample number 2 from the RSG (well 4) had 179 higher band intensity compared to sample number 1 from the RSG180 WAD cross (well 3). None of the negative control samples showed a 181 positive amplification. 
182

183

186 When the obtained PCR products were sent for sequencing, only 187 sample 2 from the RSG (product in well 4) passed the quality 188 assurance (QC) test necessary for a successful sequencing services 189 possibly because of the high intensity of the band obtained by PCR 190 amplification (Figure 2) and was therefore the only product 191 sequenced in both directions using the B2L forward and reverse 192 primers. Subsequently, the sequence obtained was subjected to 193 BLAST search in the NCBI database and the identity was confirmed 194 as ORFV which was named More strain. The sequence was aligned 195 with downloaded ORFV reference sequences at nucleotide (Figure 196 3) and amino acid levels (Figure 4) to observe for similarities and 197
Figure 2: The PCR product showing the 1137bp fragments from sample 1 (well 3) and sample 2 (well 4, More strain) with the $1 \mathrm{~kb}$ DNA ladder (well 2) and the negative control (well 5)

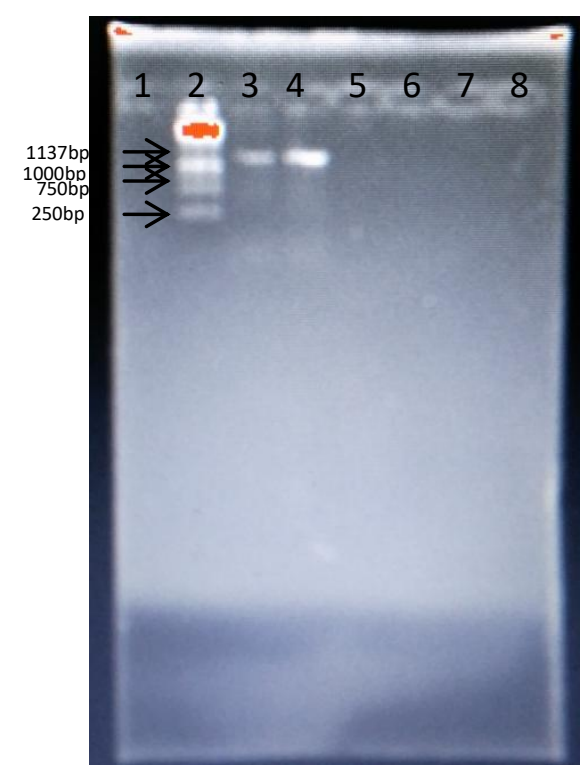




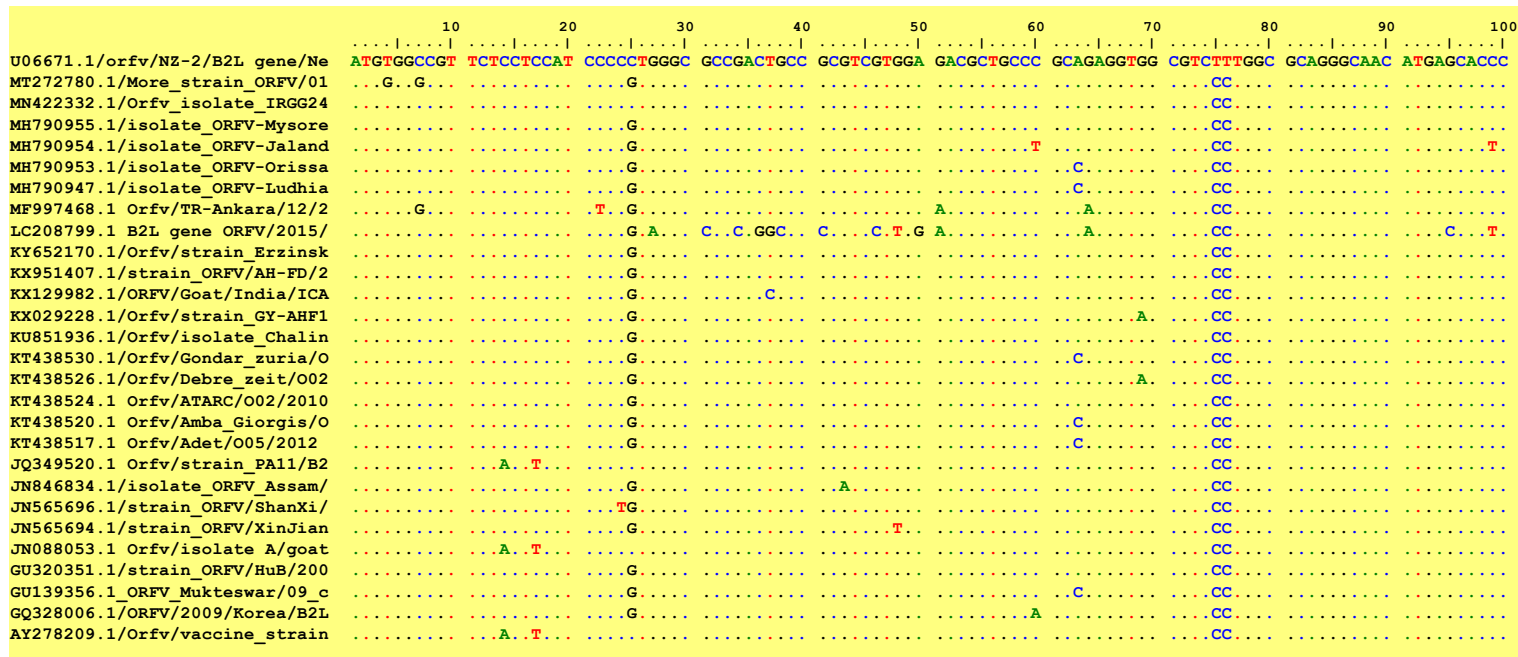

作

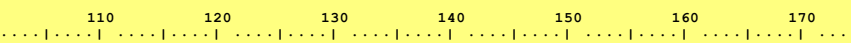

$180 \quad 190 \quad 200$

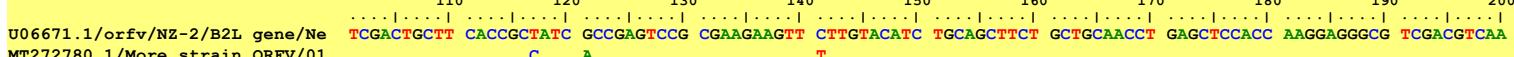
MT272780.1/More_strain_ORFV/01 MN422332.1/0rfv_isolate_IRGG24 MH790955.1/isolate_ORFV-Mysore M7. MH79953.1/1solate_ORFV-Oriss MF MF997468.1 OrfV/TR-Ankara/12/2 ry652170.1/Orfv/strain KX951407.1/strain_ORFV/AH-FD/2 KX129982. 1/ORFV/Goat/India/IC KX029228.1/Orfv/strain GY-AHF KU851936.1/Orfv/isolate_chalin KT438530.1/Orfv/Gondar_zuria/O KT438526.1/Orfv/Debre_zeit/002 KT438524.1 Orfv/ATARC/002/2010 KT438520.1 Orfv/Amba_Giorgis/ KT438517.1 Orfv/Adet/005/2012 S349520.1 Orfv/strain_PA11/B

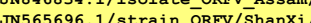
N5565594.1/stain_oRF/ JN088053 1 orfv/isolv/XinJia GU320351.1/strain ORFV/HuB/200 GU139356.1 ORFV Mukteswar/09 GQ328006.1/ORFV/2009/Korea/B2 AY278209.1/Orfv/vaccine_strain

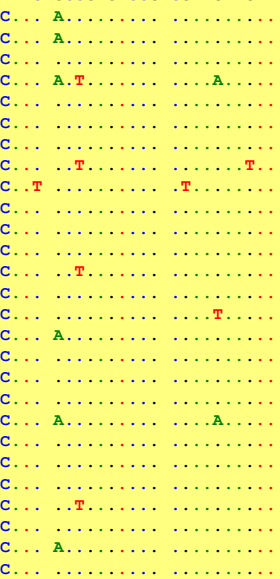

$\begin{array}{llllllllll}210 & 220 & 230 & 240 & 250 & 260 & 270 & 280 & 290 & 300\end{array}$

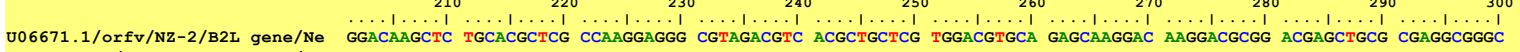
MT272780.1/More_strain ORFV/01 MN422332.1/Orfv_isolate_IRGG24 Ming MH790953.1/isolate-OPFV-Orand MH790947.1/isolate_ORV-Oriss MF997468.1 Orfv/TR-Ankara/12/2 LC208799.1 B2L gene ORFV/2015/ KY652170.1/Orf KX951407.1/strain_ORFV/AH-FD/2 KX129982.1/ORFV/Goat/India/ICA KX029228.1/Orfv/strain_GY-AHF KU851936.1/Orfv/isolate_Chalin KT438530.1/Orfv/Gondar_zuria/O KT438526.1/OrIV/Debre_zeit/O02 KT438520.1 Orfv/Ambacior (1) Je349520.1 Orfv/st /strain_PA11/B2 JN846834.1/isolate_ORFV_Assam/ JN565696.1/strain_ORFV/ShanXi/ JN088053.1 Orfv/isolate A/goa GU320351.1/strain_ORFV/HuB/200 GQ328006.1/ORFV/2009/Korea/B2I

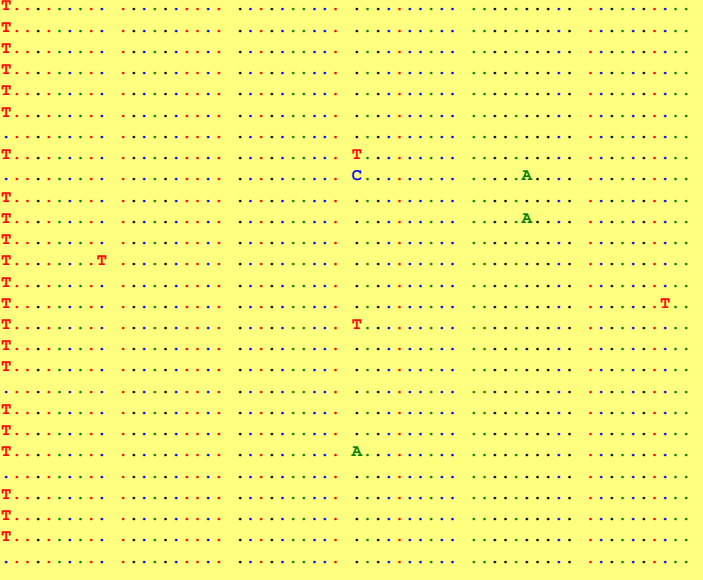
G. . c. G.

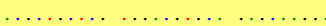

..........

c.

... G.

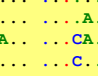

W.A.

W. . : 1/orfv/vaccine_strain 


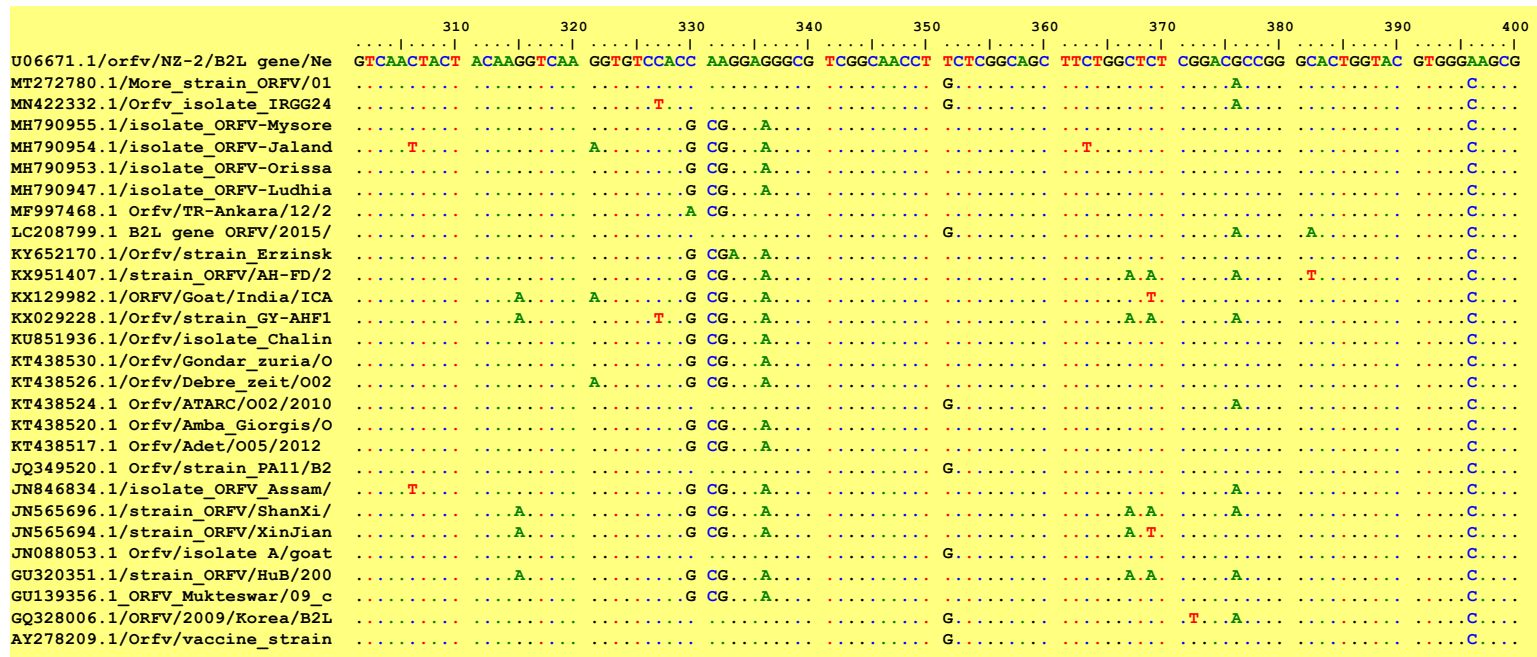

1/Orfv/vaccine strain

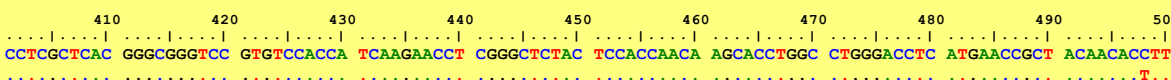

U06671.1/orfv/NZ-2/B2L gene/Ne MN422332. $1 /$ More_strain_oRF_ isolate_IRG62 MH790955.1/isolate_ORFV-Mysor MH790954.1/isolate_ORFV-Jaland MH790953.1/isolate_ORFV-Orissa MH790947.1/i solate_ORFV-Ludhia MF997468.1 OrfV/TR-Ankara/12/2 LC208799.1 B2L gene ORFV/2015/ KX951407.1/strain $\mathrm{OPFV} / \mathrm{HH}-\mathrm{FD} / 2$ KX129072. KX029228.1/Orf $\mathrm{V} / \mathrm{strain} G \mathrm{CY}-\mathrm{MHA}$ KU851936.1/Orfv/isola___CAHF KT438530.1/Orfv/Gondare_Chalin KT438526.1/Orfv/Debre zeit/O/O KT438524.1 Orfv/ATARC/002/2010 KT438520.1 Orfv/Amba_Giorgis/ KT438517.1 Orfv/Adet/005/2012 JQ349520.1 Orfv/strain_PA11/B2 JN846834.1/isolate_ORFV_Assam/ JN565696.1/strain_ORFV/ShanXi/ JN565694.1/strain_ORFV/XinJian JN088053.1 Orfv/isolate A/goat GU139356.1 OREv Murteswar/09 Co GQ328006.1/ONF/2009/Korea/B2

(

U06671.1/orfv/NZ-2/B2L gene/Ne MT272780.1/More_strain_ORFV/01 MN422332.1/Orfv_isolate_IRGG24 MH790955.1/isolate_ORFV-Mysore MH790954.1/isolate_ORFV-Jaland MH790953.1/isolate_ORFV-Oriss MH790947.1/isolate_ORFV-Ludhi MF997468.1 Orfv/TR-Ankara/12/2 LC208799.1 B2L gene ORFV/2015/ Kr652170.1/Orfv/strain_Erzinsk KX129982 1/ORFV/Got/India/ICA KX029228 1/Orfv/strain GY KX29228.1/Orf/strain_GY-AHF KT438530.1/Orfv/Gondar_Chalin KT438526.1/Orfv/Debre zeit/O02 KT438524.1 Orfv/ATARC/002/2010 KT438517.1 Orfv/Adet/005/2012 JQ349520.1 Orfv/strain_PA11/B2 JN846834.1/isolate ORFV Assan/ JN565696.1/strain_ORFV/ShanXi/ JN565694.1/strain_ORFV/XinJian JN088053.1 Orfv/isolate A/goat GU320351.1/strain_ORFV/HuB/200

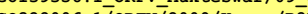
GQ328006.1/ORFV/2009/Korea/B2

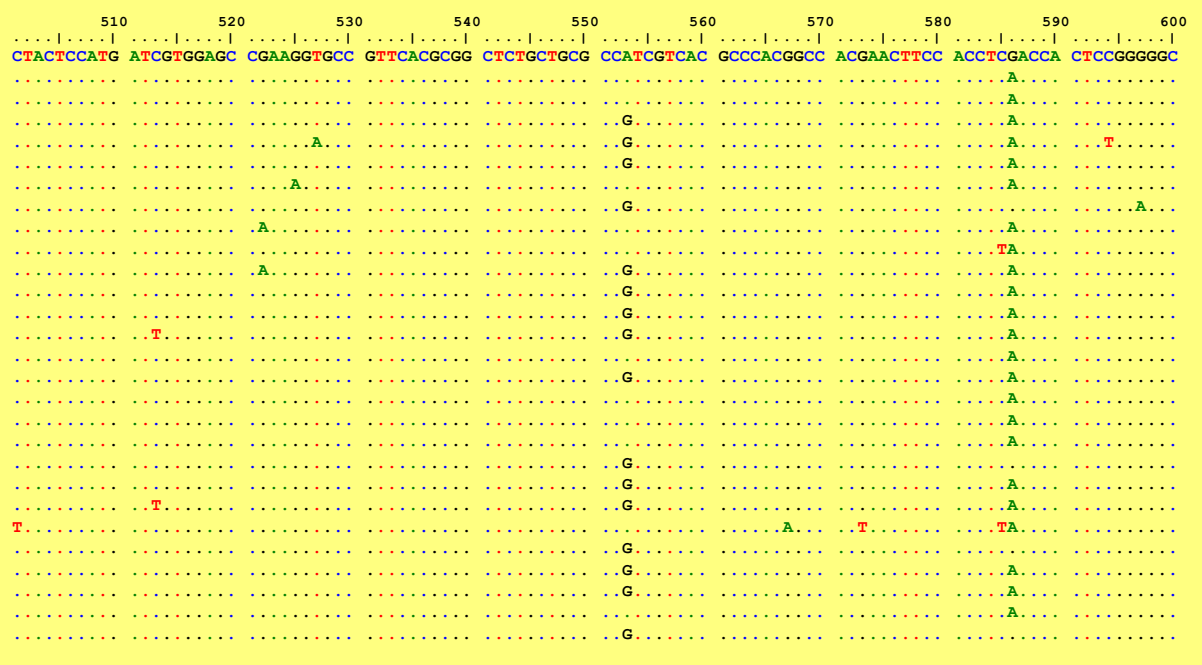




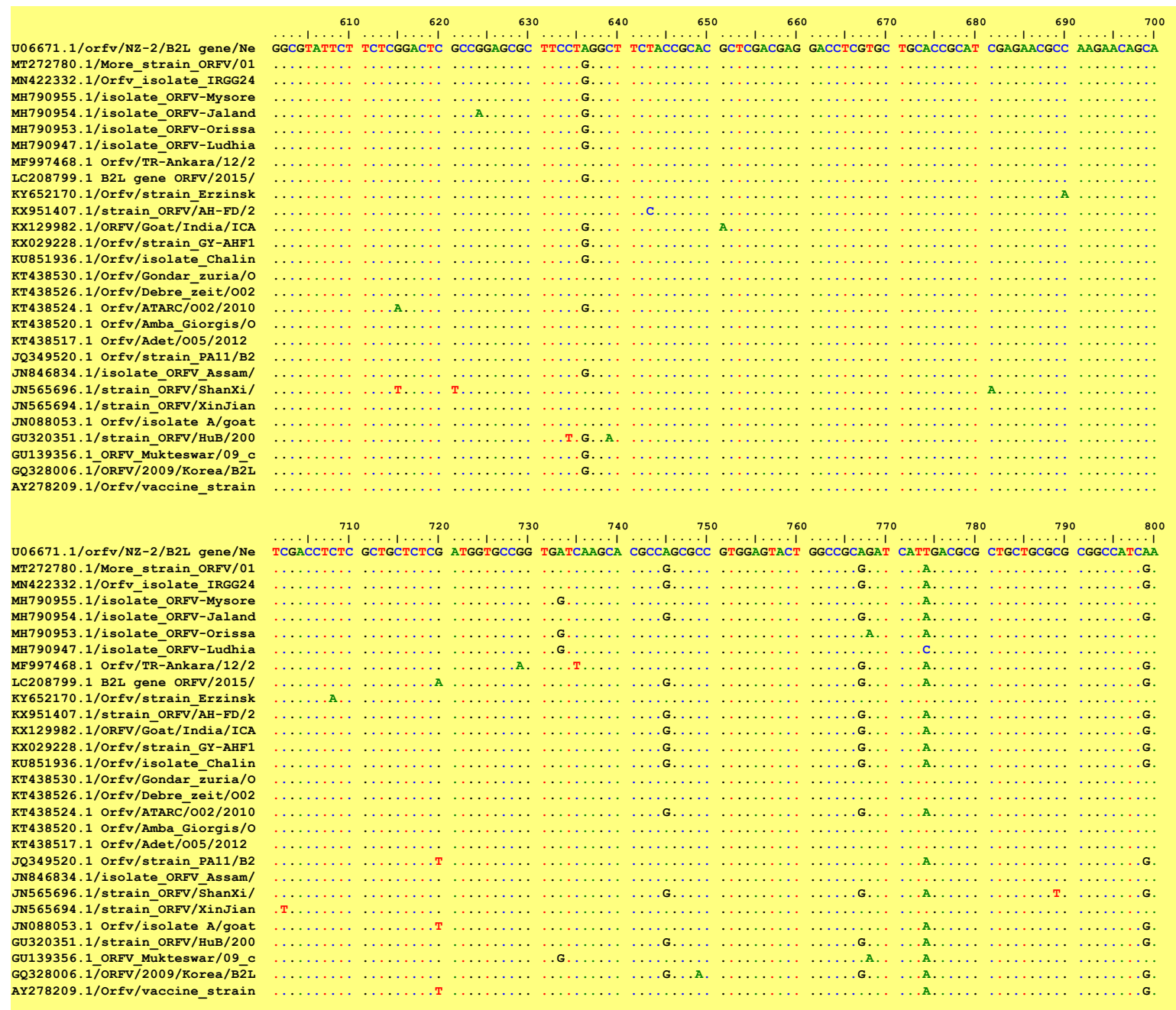

/orfv/vaccine_strai

810

820

$830 \quad 840$

850

$860 \quad 870$

$880 \quad 890 \quad 900$

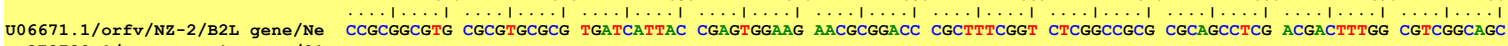
MT272780.1/More_strain_ORFV/01

MN422332.1/Orfv_isolate_IRGG2 MH790955.1/1solate_ORFV-Mysor MH790953 1/isolate_ORFV-Orissa $790947.1 /$ isolate_ORFV-Lasia MF997468.1 Orfv/TR-Ankara/12/2 LC208799. 1 B2L gene ORFV/2015/ KY652170.1/Orfv/strain Erzinsk KX951407.1/strain ORFV/AH-FD/2 KX129982.1/ORFV/Goat/India/ICA KX029228.1/Orfv/strain_GY-AHF KU851936.1/Orfv/isolate_Chali KT438530.1/Orfv/Gondar_zuria/O KT438526.1/Orfv/Debre_zeit/002 KT438524.1 OrfV/ATARC/O02/2010 KT438520.1 Orfv/Amba_Giorgis/O T230517.1 Orev/Adet/005/2012 234520.1 rev/strain_PA11/B2 J556596.1/strain OPFV/shanXi/ N5565694.1/strain_ORV/AhanXi/ JN088053.1 Orfv/isolate A/goat GU320351.1/strain_ORFV/HuB/200 GU139356.1 ORFV Mukteswar/09 GQ328006.1/ORFV/2009/Korea/B2 


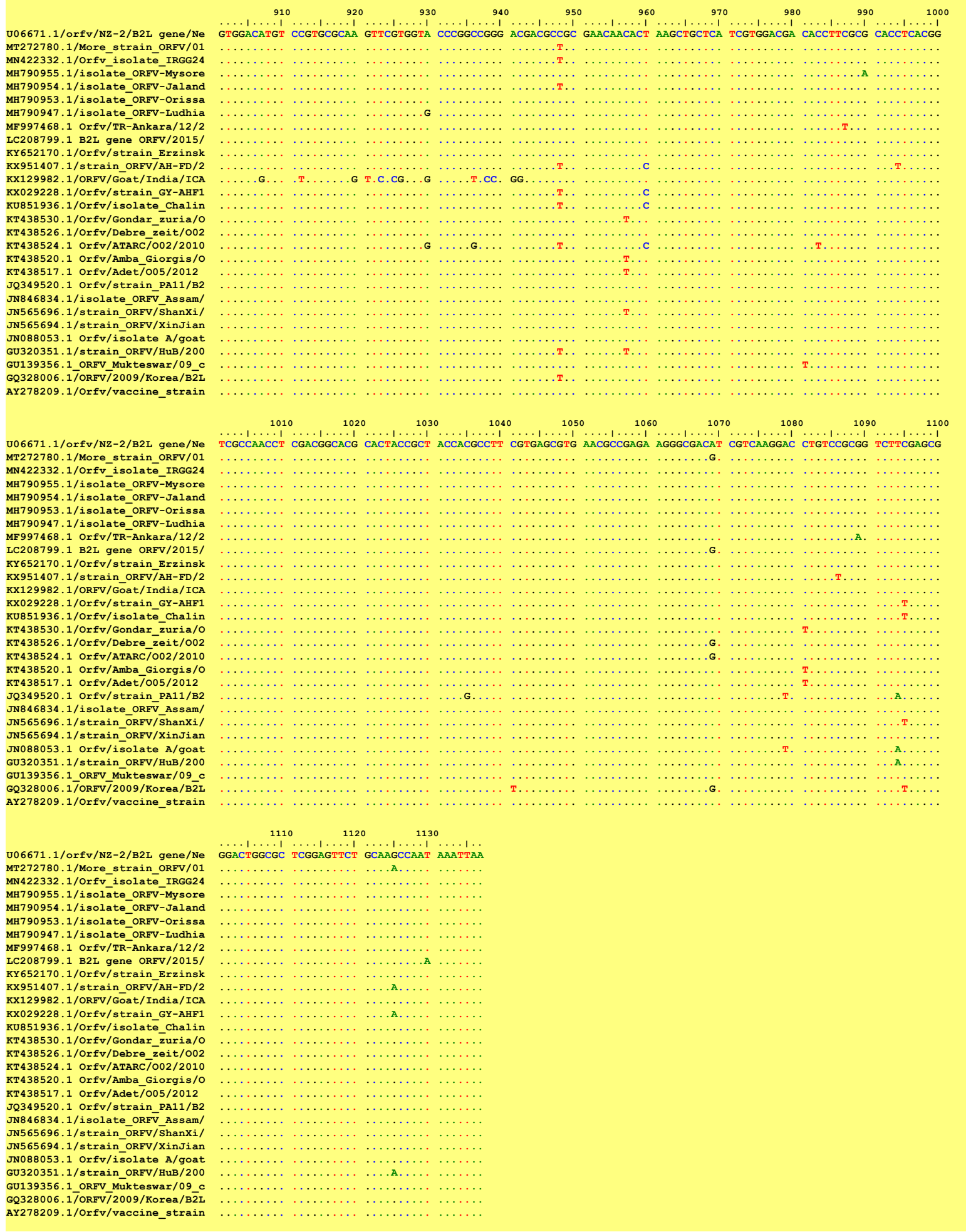

Figure 3: Nucleotide sequence comparison between More strain and reference sequences downloaded from the GeneBank, NCBI. The strain ORFV/NZ-2 from New Zealand (accession number U06671.1) was used as the guide sequence. Areas of similarity with guide sequence were represented as dots (....), while areas of differences were represented by a letter denoting the nucleotide 


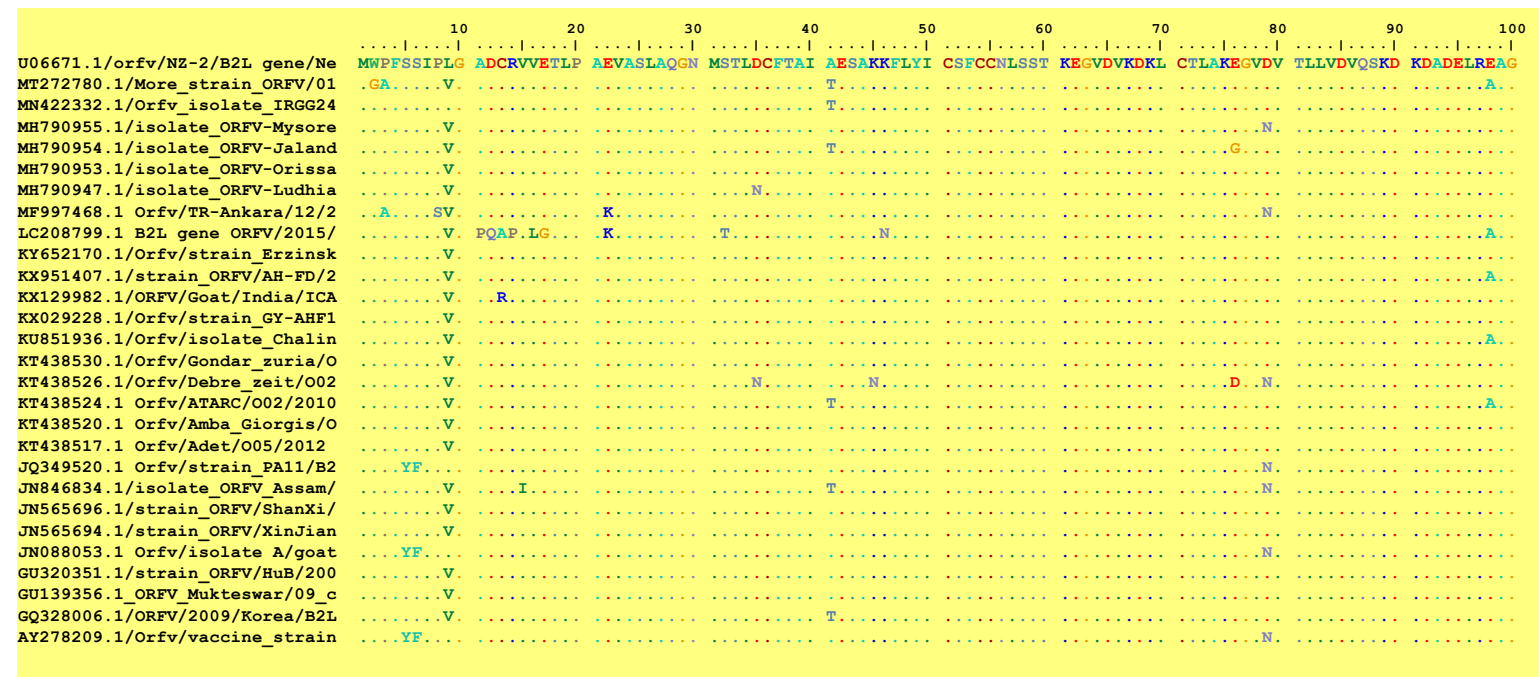

U06671.1/orfv/NZ-2/B2L gene/Ne
MT272780.1/Morestrain ORFV/01 MT272780.1/More_strain_ORFV/01 MN422332.1/Orfv_isolate_IRGG24
MH790955.1/isolate_ORFV-Mysore MH790955.1/isolate_ORFV-Mysore
MH790954.1/isolate_ORFV-Jaland MH790954.1/isolate_ORFV-Jaland MH790947.1/isolate_ORFV-Ludhia MF C208799.1 Orev/In-Ankara/12/2 KY652170 1/0rfv/strain KX951407.1/strain_OREV/AH-FD/2 KX129982.1/ORFV/Got/India/ICA KX029228.1/Orfv/strain GY-AHF1 KU851936.1/Orfv/isolate_Chal in KT438530.1/Orfv/Gondar zuria/O KT438526.1/Orfv/Debre zeit/002 KT438524.1 Orfv/ATARC/002/2010 KT438520.1 Orfv/Amba_Giorgis/O KT438517.1 Orfv/Adet/005/2012 JQ349520.1 Orfv/strain_PA11/B2 JN846834.1/1solate_OR__Assam/ JN565694.1/strain_oRp/xinXi/ JN088053.1 GU320351.1/strain ORFV/H/g/200 GU139356.1 ORFV Mukteswar/09 C GQ328006.1/ORFV/2009/Korea/B2L AY278209.1/Orfv/vaccine_strain

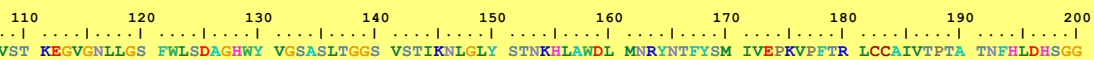

U06671.1/orfv/NZ-2/B2L gene/Ne
MT272780.1/More_strain_ORFV/01 MT272780.1/More_strain_ORFV/01
MN422332.1/Orfv_isolate_IRGG24 MH790955.1/isolate_ORFV-Mysore MH790954.1/isolate_ORFV-Jaland MH790953 1/isolate ORFV-Orissa MH790947 1/isolate-OREV-Ludhia MF997468.1 orfv/TR-Ankarad12/2 LC208799.1 B2L gene ORFV/2015/ KY652170.1/Orfv/strain Erzinsk KX951407.1/strain ORFV/AH-FD/2 KX029228.1/Orfv/strain GY-AHF1 Ku851936.1/Orfv/isolate KT438530.1/Orf//Gondar_uria/0 KT438526.1/Orfv/Debre_zeit/002 KT438524.1 Orfv/ATARC/O02/2010 KT 38520.1 Orfv/Amba_Giorgis/O J0349520.1 Orfv/strain PA11/B2 J JN565696.1/strain_ORFV/Assam/ JN565694 1/strain_ORFV/XinJian JN088053.1 Orfv/isolate A/goat GU320351.1/strain ORFV/HuB/200 G0328006.110RFV/2009/Korea/B2I

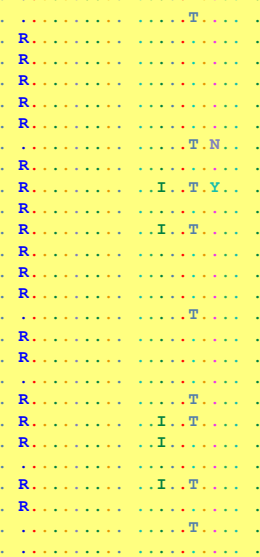

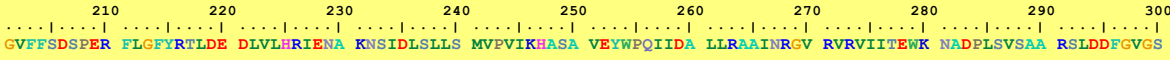

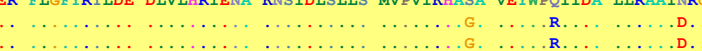

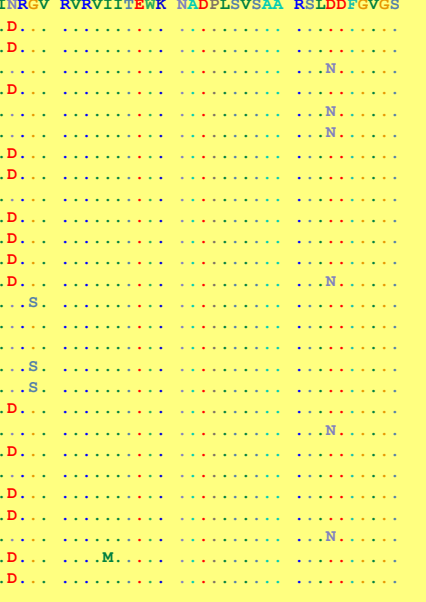




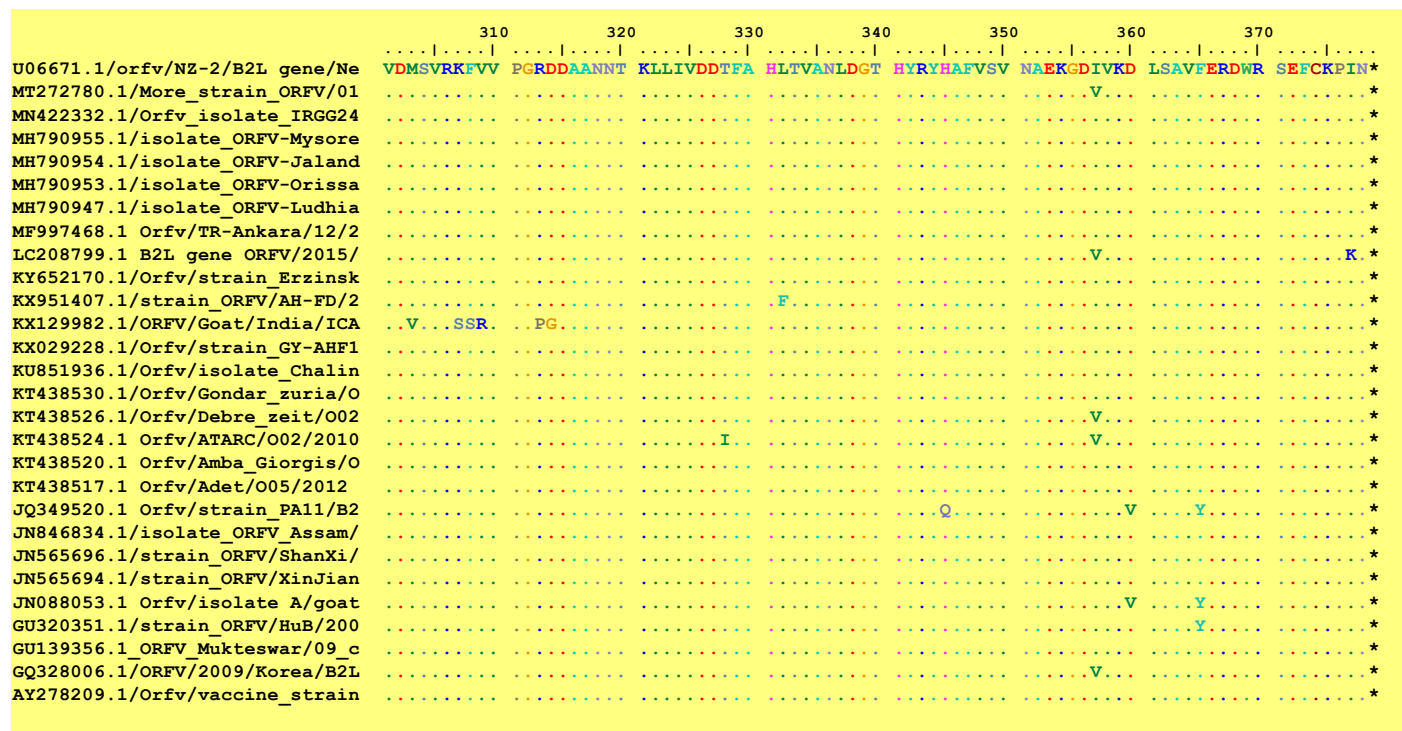

Figure 4: Amino acid sequence comparison between More strain and reference sequences downloaded from the GeneBank, NCBI. The strain ORFV/NZ-2 from New Zealand (accession number U06671.1) was used as the guide sequence. Areas of similarity with guide sequence were represented as dots (....), while areas of differences were represented by a letter denoting the amino acid

210 Comparison of the obtained sequence with reference sequences from different countries revealed nucleotide similarities range of $96.7-99.0 \%$ among the sequences.

212 Similarly, at the amino acid level, the percentage homologies ranged from 95.7-98.9\% between our isolate and the reference sequences. At the amino acid level, several mutations were observed in the More strain compared to the New Zealand NZ-2 strain used as a reference guide. There was a W2G at position 2 that was unique to the isolate studied, P3A present only in Ankara strain (MF997468), L9V present in

217 almost all the other reference sequences, A41T which More strain share with 5 other reference sequences (MN422332; MH790954; KT438524; JN846834 \& CQ328006), 219 E98A found in 4 other reference sequences (LC208799; KX951407; KU851936 \&KT438524) and A126T found also in 9 other reference sequences (MN422332; 
present in More strain and all other reference sequences except few (MF997468;

JQ349520; JN088053 and the vaccine strain AY278209), S249G, Q256R, N267D and

finally I352V (Figure 4).

Phylogenetic tree analysis based on B2L gene showed that More strain was closely

227 related to isolate from Ethiopia (KT438524) which explained the high degree of

between them. They form a distinct cluster together with the isolates from Iran

strain was the most recent common ancestor to the Korean, Zambian, Ethiopian and

More strains. Other isolates from diverse geographical locations (Asia, Africa,

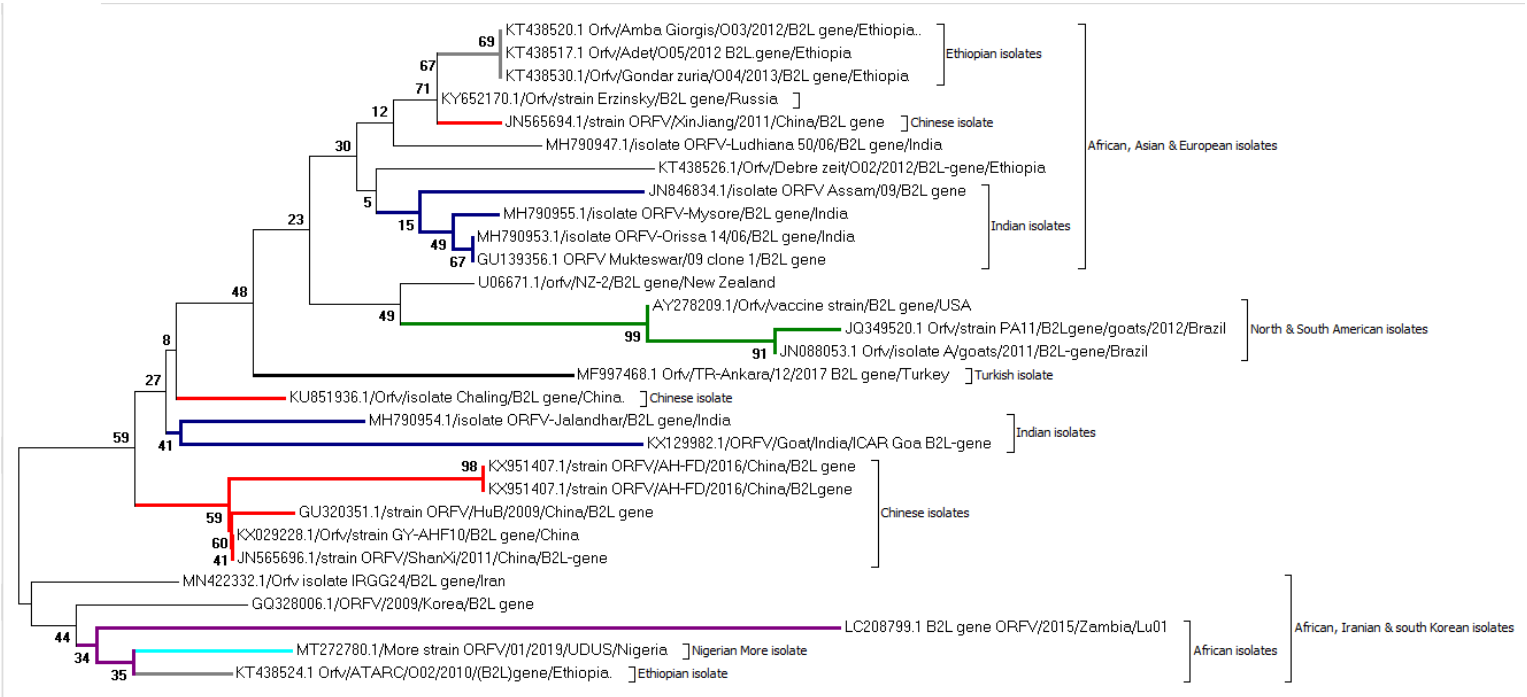

The evolutionary history was inferred using the Neighbor-Joining method. The percentage of replicate trees in which the associated taxa clustered together in the bootstrap test (2000 replicates) are shown next to the branches. The evolutionary distances were computed using the Poisson correction method and are in the units of the number of amino acid substitutions per site. The analysis involved 19 amino acid sequences. All positions containing gaps and missing data were eliminated. There were a total of 378 positions in the final dataset. Evolutionary analyses were conducted in MEGA7. 


\section{Discussion}

239 Orf or contagious ecthyma is endemic in Nigeria and causes huge

240 economic losses in the National livestock industry (4). Effective

241 control of the disease therefore demands early detection and

242 identification of the causative agent. In Nigeria, outbreaks of CE are

243 largely documented based on clinical signs which unfortunately

244 may be confused with infection resulting from other viral disease

245 agents such as sheep poxvirus, foot and mouth disease virus

246 (FMD), bovine herpes virus type-2 and bluetongue virus. Thus,

247 reliable estimate of the true prevalence of the disease in the country

248 is currently lacking. In recent times, different techniques such as

249 physical examination for clinical signs and PCR have been used to

250 confirm contagious ecthyma outbreaks in Nigeria $(1,2,4)$.

251 Furthermore, previous study conducted in 2018 involving 3 flocks

252 of goats in northern Nigeria reported a mortality rate of $100 \%$ (4) in

253 two out of the 3 flocks investigated, which was higher than usually

254 observed in CE outbreaks. This necessitated the need to

255 molecularly characterise the ORFV circulating within the northern

256 region of Nigeria to see how the Nigerian isolates may differ with

257 other isolates globally. Moreover, to the best of our knowledge, no

258 available literatures that molecularly describe the genetic

259 characteristic of ORFV circulating in any part of Nigeria exist as at

260 the time of this study. Consequently, the present study was 
261 designed to isolate and genetically characterise the virus obtained 262 from an outbreak involving a goat farm in Sokoto metropolis, 263 North Western Nigeria.

264 Between May and September 2019, a number of suspected CE 265 outbreaks have occurred in Sokoto State, Nigeria. One of those 266 outbreaks involved a goat farm located in More village within 267 Sokoto metropolis. Scabs were obtained from the two affected 268 animals and prepared for inoculation into chicken embryonated 269 eggs via the CAM route for virus isolation. Expectedly, the CAM 270 developed characteristic pock lesions typical of Orf viruses, 271 signifying virus replication. Although this method of virus

272 isolation is simple and relatively rapid for Orf diagnosis, to the best

273 of our knowledge, this is the first report of Orf virus isolation using

274 specific antibody free chicken embryonated eggs in Nigeria.

275 Full length B2L gene fragments were obtained by PCR 276 amplification and the full length sequence of one of the samples 277 (sample 2) was obtained from Inqaba, South Africa. The other 278 sample 1 was not sequenced because the PCR product failed the QC 279 necessary for a successful sequencing. The sequence obtained 280 confirmed the identity of the virus isolate as ORFV when blasted 281 using the NCBI BLASTN tool. The virus when aligned with 282 deposited ORFV sequences downloaded from NCBI database 283 showed similarities with the reference sequences at both nucleotide 
284 and amino acid levels. A unique amino acid change observed only 285 in the strain under study was the W2G seen at amino acid position

286 2. Another mutation observed was the P3A change at position 3 287 that was seen only in the Ankara strain (MF997468) from Turkey.

288 However, the significance of these two substitution mutations as 289 they affect the virulence and pathogenicity of the ORFV isolate 290 under study need to be investigated.

291 Phylogenetic tree analysis based on the B2L gene showed two 292 major branches on the tree. On the first branch, the IRGG24 293 (MN422332) isolate from Iran was placed at the root of this branch 294 indicating that it is the most recent common ancestor from which 295 other ORFV isolated from Korea (GQ328006), Zambia (LC208799), 296 Ethiopia (KT438524) and the isolate under study originated. The 297 position of the More strain (light green line) on this branch placed it 298 more closely with the Ethiopian isolate (KT438524) which explained 299 the high degree of nucleotide and amino acid similarities of $99.0 \%$ 300 and $98.9 \%$ respectively that exist between the two isolates. The 301 second branch on the tree had four major sub-branches where 302 Chinese isolates (red line) diverged from the others, while the New 303 Zealand (U06671), USA vaccine (AY278209) and two Brazilian 304 strains (JQ349520 \& JN088053) clustered together (green line) in a 305 separate branch. Isolates from India (blue line) clustered together 306 with one Ethiopian strain in a separate sub-branch. Finally, three 
307 Ethiopian isolates clustered with Erzinsky strain (KY652170) from

308 Russia and XinJiang strain (JN565694) from China in a separate sub309 branch. This analysis indicated that the Nigerian isolate 310 investigated is genetically different with other ORFV isolated from 311 different geographic regions of the world. However, the 312 significance of this finding can only be fully understood when 313 investigated further.

\section{Conclusion}

315 In summary, we have examined the genetic characteristics of ORFV 316 causing disease outbreaks in Sokoto Metropolis, Nigeria and 317 provided molecular signatures of the virus in the study area. We 318 showed that the virus shared a close genetic relationship with an 319 isolate from Ethiopia and harbours an amino acid change (W2G) 320 that is unique to it and another mutation at P3A that is present in 321 only in the Ankara strain (MF997468). This information is crucial to

322 understand the molecular epidemiology of ORFV circulating in 323 Nigeria, which is necessary for the design of effective CE vaccines 324 which are currently lacking in the country.

\section{Future Perspective}

326 There is the need to undertake a state wide isolation and characterization study to 327 fully understand the molecular epidemiology of the ORFV isolates circulating within 328 the goat and sheep population in Nigeria. Whole genome sequencing can be done to 329 add to the existing ORFV complete genome sequences since, currently very few are 330 in the public database. The significance of the W2G and P3A mutations with respect 331 to ORFV virulence modulation and pathogenicity needs to be investigated. 


\section{Financial and Competing Interests Disclosure}

333 The authors have no competing interest or financial involvement with any entity or 334 organization with a financial interest in or financial conflict with the subject matter 335 or materials discussed in the manuscript apart from those already disclosed.

Acknowledgements

The authors acknowledged the contributions of the entire technical staff of the Central Veterinary Research Laboratory, Faculty of Veterinary Medicine, Usmanu Danfodiyo University, Sokoto for their help in sample analysis.

\section{Summary Points}

- Scab lesions from goats suspected to be infected with ORFV based on clinical signs were obtained.

- Genomic DNA was extracted and amplified by polymerase chain reaction.

- The PCR products were analyzed by gel electrophoresis and sent for sequencing.

- The sequencing result was analyzed and aligned using ClustalW to determine its similarity with reference sequences downloaded from GeneBank.

- Deduced amino acid was determined and any change between the isolate and the reference sequences were noted.

- Phylogenetic tree was constructed to determine the taxonomic relationship between taxa.

- One unique amino acid change (W2G) and another (P3A) present only in a Turkish isolate were observed in the isolate under study.

- Phylogenetically, the isolate is more closely related to the Ethiopian isolate (KT438524) with which it cluster in the same clade different from other reference sequences.

- This is the first report on the molecular characterization of ORFV using full length B2L gene sequences.

1. Adedeji AJ, Maurice NA, Wungak YS, Adole JA, Chima NC, Woma TY, et al. 
Diagnosis of orf in west African dwarf goats in Uyo, Akwa Ibom state, Nigeria. African J Infect Dis. 2017;11(2):90-4.

2. Adedeji AJ, Gamawa AA, Chima NC, Ahmed AI, Ifende VI, Adole JA, et al. First report of camel contagious ecthyma in Nigeria. Open Vet J. 2018;8(2):208.

3. Olivero N, Reolon E, Arbiza J, Berois M. Genetic diversity of Orf virus isolated from sheep in Uruguay. Arch Virol [Internet]. 2018;(0123456789). Available from: https:/ / doi.org/10.1007/s00705-018-3717-x

4. Adedeji AJ, Adole JA, Chima NC, Maguda AS, Dyek DY, Jambol AR, et al. Contagious ecthyma in three flocks of goats in Jos-south LGA, Plateau State, Nigeria. Sokoto J Vet Sci. 2018;16(1):107.

5. Yang H, Meng Q, Qiao J, Peng Y, Xie K, Liu Y, et al. Short Communication Detection of genetic variations in Orf virus isolates epidemic in. 2014;1-6.

6. Wang G, Wang Y, Kong J, Li Y, Wu J, Chen Y. Comparison of the sensitivity of three cell cultures to ORFV. 2019;(August 2005):4-11.

7. Bala AJ, BalaKrishnan NK, Firdaus F, Jesse A, Ahmed A, Syaafii M, et al. Identification of strain diversity and phylogenetic analysis based on two major essential proteins of Orf viruses isolated from several clinical cases reported in Malaysia. Infect Genet Evol [Internet]. 2020;77(September 2019):104076. Available from: https:/ / doi.org/10.1016/j.meegid.2019.104076

8. Tedla M, Berhan N, Molla W, Temesgen W, Alemu S. Molecular identification and investigations of contagious ecthyma (Orf virus) in small ruminants, North west Ethiopia. BMC Vet Res. 2018;14(1):1-8.

9. Mazur C, Machado RD. Detection of contagious pustular dermatitis virus of goats in a severe outbreak. Vet Rec [Internet]. 1989 Oct 14;125(16):419 LP - 420. Available from: http:/ / veterinaryrecord.bmj.com/content/125/16/419.abstract

10. Bayindir Y, Bayraktar M, Karadag N, Ozcan H, Kayabas U, Otlu B, et al. Investigation and analysis of a human orf outbreak among people living on the same farm. New Microbiol. 2011;34(1):37-43.

11. Haddock Ellen S, Cheng E Carol, Bradley S John, Hsu H Christopher, Zhao Hui DBW and BRV. Extensive orf infection in a toddler with associated id reaction. Pediatr Dermatol. 2017;34(6):e337-40.

12. Nagarajan G, Pourouchottamane R, Reddy GBM, Yogisharadhya R, Sumana K, Rajapandi S, et al. Molecular characterization of Orf virus isolates from Kodai hills , Tamil Nadu , India. 2019;12.

13. Chan KW, Lin JW, Lee SH, Liao CJ, Tsai MC, Hsu WL, et al. Identification and phylogenetic analysis of orf virus from goats in Taiwan. Virus Genes. 2007;35(3):705-12. 
401

402

403

404

405

406

407

408

409

410

411

412

413

414

415

416

417

418

419

420

421

422

423

424

425

426

427

428

429

430

431

432

433

434

435

436

437

438

14. Riccardo Wittek, Clive C Kuenzle RW. High C + G Content in Parapoxvirus I ) N A. J Gen Virol. 1979;43:231-4.

15. Fleming SB, Wise LM, Mercer AA. Molecular Genetic Analysis of Orf Virus: A Poxvirus That Has Adapted to Skin. Viruses. 2015;7:1505-39.

16. Bala JA, Balakrishnan KN, Abdullah AA, Kimmy T, Abba Y, Bin Mohamed R, et al. Dermatopathology of Orf Virus (Malaysian Isolates) in Mice

Experimentally Inoculated at Different Sites with and without Dexamethasone Administration. J Pathog. 2018;2018:1-12.

17. Gelaye E, Achenbach JE, Jenberie S, Ayelet G, Belay A. Molecular characterization of orf virus from sheep and goats in Ethiopia , 2008 - 2013. Virol J [Internet]. 2016;(February):2008-13. Available from: http:/ / dx.doi.org/10.1186/s12985-016-0489-3

18. Hosamani M, Scagliarini A, Bhanuprakash V, McInnes CJ, Singh RK. Orf: an update on current research and future perspectives. Expert Rev Anti Infect Ther [Internet]. 2009 Sep 1;7(7):879-93. Available from: https:/ / doi.org/10.1586/ eri.09.64

19. Zhang K, Lu Z, Shang Y, Zheng H, Jin Y, He J, et al. Diagnosis and phylogenetic analysis of Orf virus from goats in China: A case report. Virol J. 2010;7(April).

20. Zhang K, Liu Y, Kong H. Comparison and phylogenetic analysis based on the B2L gene of orf virus from goats and sheep in China during 2009-2011. 2014;2011:1475-9.

21. Mwanandota JJ, Macharia M, Sallu R, Yongolo M, Holton TA. Phylogenetic Analysis of ORF Virus from Goats in Tanzania : Short Communication. 2016;4(5):165-9.

22. Ferede Y, Habtamu A, Gebresellasie S. Animal Health Confirmatory diagnosis of contagious ecthyma (Orf) by polymerase chain reaction at Adet Sheep Research Sub-Center, Ethiopia : A case report. 2014;6(July):187-91.

23. Mahmoud M, Soliman H. Molecular and virological studies on contagious pustular dermatitis isolates from Egyptian sheep and goats. 2010;(March).

24. Zeedan GSG, Abdalhamed AM, Ghoneim NH, Ghazy AA. Antivirals \& Antiretrovirals Isolation and Molecular Diagnosis of Orf Virus from Small Ruminants and. 2015;7(1):2-9.

25. Maganga GD, Relmy A, Bakkali-kassimi L, Ngoubangoye B, Tsoumbou T, Bouchier C, et al. Molecular characterization of Orf virus in goats in Gabon, Central Africa. Virol J [Internet]. 2016;1-5. Available from: http:/ / dx.doi.org/10.1186/s12985-016-0535-1

26. Khalafalla AI, El-sabagh IM, Al-busada KA, Al-mubarak AI, Ali YH. 
Phylogenetic analysis of eight sudanese camel contagious ecthyma viruses based on B2L gene sequence. Virol J [Internet]. 2015;1-9. Available from: http://dx.doi.org/10.1186/s12985-015-0348-7

27. Ibrahim A, Eisa A, Zackaria H, Ishag A. Field investigation and phylogenetic characterization of orf virus ( ORFV) circulating in small ruminants and Pseudocowpoxvirus ( PCPV) in dromedary camels of eastern Sudan. Heliyon [Internet]. 2020;6(November 2019):e03595. Available from: https://doi.org/10.1016/j.heliyon.2020.e03595

28. Lawal N, Hair-Bejo M, Arshad SS, Omar AR, Ideris A. Adaptation and Molecular Characterization of Two Malaysian Very Virulent Infectious Bursal Disease Virus Isolates Adapted in BGM-70 Cell Line. Adv Virol [Internet]. 2017;2017:1-19. Available from: https://www.hindawi.com/journals/av/2017/8359047/

29. Hosamani M, Bhanuprakash V, Scagliarini A, Singh RK. Comparative sequence analysis of major envelope protein gene (B2L) of Indian orf viruses isolated from sheep and goats. Vet Microbiol [Internet]. 2006;116(4):317-24. Available from: http://www.sciencedirect.com/science/article/pii/S0378113506001829

30. Kumar S, Stecher G, Tamura K. MEGA7: Molecular Evolutionary Genetics Analysis Version 7.0 for Bigger Datasets. Mol Biol Evol [Internet]. 2016;33(7):1870-4. Available from: https://academic.oup.com/mbe/articlelookup/doi/10.1093/molbev/msw054 